\title{
EFFECTS OF NON-ISOTROPIC SCATTERING, MAGNETIC HELICITY, AND ADIABATIC FOCUSING ON DIFFUSIVE TRANSPORT OF SOLAR ENERGETIC PARTICLES
}

\author{
YURI E. LITVINENKO \\ Department of Mathematics, University of Waikato, P. B. 3105, Hamilton, New Zealand \\ Received 2012 February 15; accepted 2012 March 30; published 2012 May 22
}

\begin{abstract}
Transport of solar energetic particles in interplanetary space is analyzed. A new systematic derivation of the diffusion approximation is given, which incorporates the effects of non-isotropic scattering, magnetic helicity, and adiabatic focusing in a non-uniform large-scale magnetic field. The derivation is based on a system of stochastic differential equations, equivalent to the Fokker-Planck equation, and the new method is a generalization of the Smoluchowski approximation in the theory of the Brownian motion. Simple, physically transparent expressions for the transport coefficients are derived. Different results of earlier treatments of the problem are related to the assumptions regarding the evolving particle distribution.
\end{abstract}

Key words: diffusion - magnetic fields - scattering - Sun: particle emission

\section{INTRODUCTION}

When energetic particles propagate in interplanetary space following large solar flares, the particles interact with a turbulent interplanetary magnetic field. The resulting particle transport is diffusive (Roelof 1969). A diffusion equation for the particle density is known to approximate the Fokker-Planck equation when the particle pitch-angle distribution is weakly anisotropic, in which case the scale of density variation is larger than the scattering mean free path (Jokipii 1966; Hasselmann \& Wibberenz 1968, 1970; Schlickeiser \& Shalchi 2008, and references therein).

Early theoretical models neglected the coherent propagation of flare-accelerated particles and attempted to describe the observed particle spectra using solutions of the spatial diffusion equation with an empirical diffusion coefficient (Meyer et al. 1956). More detailed physical models were later developed which described diffusive transport of cosmic-ray particles, taking into account the effects of coherent propagation, magnetic helicity, and adiabatic focusing in a non-uniform mean magnetic field (Earl 1981; Beeck \& Wibberenz 1986; Bieber et al. 1987; Bieber \& Burger 1990; Kóta 2000; Schlickeiser \& Shalchi 2008). Solar cosmic-ray data have been analyzed in terms of focused particle transport in interplanetary space (e.g., Bieber et al. 2002; Sáiz et al. 2008). Notably, theoretical predictions of the focused transport model appear to be in reasonable agreement with the flare electron spectra obtained with the Wind spacecraft (Artmann et al. 2011).

The value of the coefficient $\kappa_{\|}$for spatial diffusion parallel to the mean magnetic field is of particular importance in practical applications of diffusive transport models. Previously, the value of $\kappa_{\|}$has been calculated by several independent methods: the parallel streaming inferred from an exact steady solution to the Fokker-Planck equation (Earl 1981; Beeck \& Wibberenz 1986), the Born approximation (Bieber \& Burger 1990), and the adjoint Green's function technique (Kóta 2000). The calculations agree with one another if a pitch-angle scattering coefficient in the Fokker-Planck equation is an even function of the cosine $\mu$ of the pitch angle (for a recent discussion see Litvinenko 2012). A non-zero magnetic helicity, however, leads to a pitch-angle scattering coefficient that is not an even function of $\mu$. Bieber et al. (1987) argued that the general expression for $\kappa_{\|}$, originally derived by Beeck \& Wibberenz (1986), can also be used when a helicity parameter $\sigma \neq 0$. By contrast Bieber \& Burger (1990) and Kóta (2000) gave different expressions for $\kappa_{\|}$which reduce to that in Beeck \& Wibberenz (1986) only if $\sigma=0$. The seemingly conflicting theoretical results make it worthwhile to revisit both the calculation of $\kappa_{\|}$and the assumptions on which the diffusion approximation is based.

Motivated by these considerations, the purpose of this paper is to give a new systematic derivation of the diffusive limit of cosmic-ray particle transport, which incorporates the effects of non-isotropic scattering, magnetic helicity, and adiabatic focusing. The derivation is based on the fact that the Fokker-Planck equation for particle transport is completely equivalent to a system of stochastic differential equations. The equations are easy to analyze when strong pitch-angle scattering causes the particle angular distribution to relax rapidly to a near-isotropic state. A stochastic method has been recently applied to focused transport with isotropic scattering and vanishing helicity (Litvinenko 2012). The present paper generalizes the method to a helicity-dependent pitch-angle diffusion coefficient in the Fokker-Planck equation and clarifies the dependence of $\kappa_{\|}$on magnetic helicity. The new description brings out the key assumptions of the diffusion approximation and explains the differences in the previously derived expressions for the parallel diffusion coefficient.

\section{THE FOKKER-PLANCK AND STOCHASTIC DESCRIPTIONS OF PARTICLE TRANSPORT}

The evolution of a cosmic-ray particle distribution is described by the Fokker-Planck equation that incorporates the effects of pitch-angle scattering and adiabatic focusing:

$$
\frac{\partial f_{0}}{\partial t}+\mu v \frac{\partial f_{0}}{\partial z}+\frac{v}{2 L}\left(1-\mu^{2}\right) \frac{\partial f_{0}}{\partial \mu}=\frac{\partial}{\partial \mu}\left(D_{\mu \mu} \frac{\partial f_{0}}{\partial \mu}\right)
$$

(e.g., Roelof 1969; Earl 1981; Schlickeiser 2011). Here $f_{0}$ is the distribution function of energetic particles (gyrotropic phase-space density), $t$ is time, $\mu$ is the cosine of the particle pitch angle, $v$ is the particle speed, $z$ is the distance along the mean magnetic field $B_{0}, L=-B_{0} /\left(\partial B_{0} / \partial z\right)$ is the adiabatic focusing length ( $L=$ constant is assumed below), and $D_{\mu \mu}$ is the Fokker-Planck coefficient for pitch-angle scattering. Momentum diffusion can be neglected for the transport of solar energetic particles (Artmann et al. 2011). 
The scattering coefficient $D_{\mu \mu}$ due to resonant interaction of charged particles and magnetohydrodynamic turbulence is a function of the magnetic and cross helicities of the turbulence. In a slab model of Alfvénic turbulence, the cross helicity $H_{c}$ is defined by the relative intensities of forward- and backwardpropagating waves:

$$
H_{c}=\frac{I^{+}-I^{-}}{I^{+}+I^{-}} .
$$

Here $I^{+}=I_{L}^{+}+I_{R}^{+}$is the intensity of the forward-propagating waves, $I^{-}=I_{L}^{-}+I_{R}^{-}$is the intensity of the backwardpropagating waves, and $L$ and $R$ indicate the left- and right-handed wave polarizations. The magnetic helicity is characterized by the polarization parameters:

$$
\sigma^{+}=\frac{I_{L}^{+}-I_{R}^{+}}{I_{L}^{+}+I_{R}^{+}}, \quad \sigma^{-}=\frac{I_{L}^{-}-I_{R}^{-}}{I_{L}^{-}+I_{R}^{-}} .
$$

A concrete example, often considered in the literature on diffusive particle transport, is that of isospectral Alfvénic slab turbulence with constant magnetic and cross helicities that are independent of the wave number (Dung \& Schlickeiser 1990, and references therein). If there are only forward-propagating waves of the same phase speed, then $I^{-}=0, H_{c}=1, \sigma^{-}=0$, and $\sigma^{+}=\sigma=$ constant. The polarization parameter $\sigma$ can vary from -1 (pure right-hand circularly polarized turbulence) to +1 (pure left-hand circularly polarized turbulence). The corresponding helicity-modified scattering coefficient for the slab model of turbulence is given by

$$
D_{\mu \mu}=D_{0}\left(1-\mu^{2}\right)|\mu|^{q-1}\left[1+\sigma \operatorname{sgn}\left(e B_{0} \mu\right)\right]
$$

(Goldstein \& Matthaeus 1981). Here $D_{0}=$ constant, $e$ is the particle charge, and $q=$ constant specifies the anisotropy of scattering. Physically, $q$ is the spectral index of the magnetic power spectrum in inertial range. Following the previous treatments of the problem (e.g., Bieber et al. 1987; Bieber \& Burger 1990; Kóta 2000), this expression for $D_{\mu \mu}$ is used in the analysis below (for more general formulas see Schlickeiser 1989; Dung \& Schlickeiser 1990). For simplicity, $e B_{0}>0$ is assumed, so that the sign function $\operatorname{sgn}\left(e B_{0} \mu\right)=\operatorname{sgn}(\mu)$. The spectral index is assumed to be in the range $1 \leqslant q<2$.

The Fokker-Planck equation is equivalent to a system of stochastic differential equations (e.g., Gardiner 2009). It often turns out to be useful to consider the stochastic equations in both numerical (e.g., Fichtner et al. 1996; Zhang 1999; Pei et al. 2010; Strauss et al. 2011) and analytical (Conway et al. 1998; Conway 2000; Litvinenko 2009) treatments of various problems of cosmic-ray particle transport. When Equation (1) is rewritten in terms of the linear density $f=\exp (z / L) f_{0}$, application of the Itô calculus leads to the following stochastic system:

$$
\begin{gathered}
d z=\mu v d t \\
d \mu=\left[\frac{v}{2 L}\left(1-\mu^{2}\right)+\frac{\partial D_{\mu \mu}}{\partial \mu}\right] d t+\sqrt{2 D_{\mu \mu}} d W
\end{gathered}
$$

(Litvinenko 2012). Here $W(t)$ represents a Wiener process with zero mean and variance equal to $t$. Equations (5) and (6) contain the same information about the evolution of the particle distribution function as Equation (1).

Since Equation (6) does not contain $z$, it can be integrated to give an exact steady anisotropic distribution $f(z, \mu)=h(\mu)$, where

$$
h(\mu)=\text { constant } \times e^{G}
$$

and

$$
G(\mu)=\frac{v}{2 L} \int_{0}^{\mu} \frac{1-\mu^{\prime 2}}{D_{\mu \mu}\left(\mu^{\prime}\right)} d \mu^{\prime}
$$

(Beeck \& Wibberenz 1986).

The analysis so far is exact. The next section presents a derivation of the diffusion approximation for the evolution of the particle density. The approximation is valid when strong scattering causes the time-dependent angular distribution of energetic particles to remain close to the steady distribution $h(\mu)$.

\section{THE DIFFUSION APPROXIMATION}

When a characteristic evolution timescale of the particle distribution is much greater than the pitch-angle scattering time, the initial angular distribution relaxes rapidly to a state that is close to the exact steady solution given by Equation (7). Hence it seems possible to set $d \mu \approx 0$ in the stochastic Equation (6) and use the resulting equation to calculate the distribution anisotropy and the corresponding spatial diffusion coefficient. The approach is analogous to the Smoluchowski approximation to the Langevin equation, which leads to the usual diffusion equation for the Brownian motion. Strong angular scattering implies that $\mu$ is a fast variable that can be adiabatically eliminated (e.g., chapter 8 in Gardiner 2009).

Litvinenko (2012) used this argument and set $d \mu=0$ to study a diffusive limit of the particle evolution in the case of isotropic $(q=1)$ scattering in the absence of net polarization $(\sigma=0)$. Generalizing the argument for an arbitrary $q$ is not straightforward, however, because the right-hand side of Equation (6) contains a term $\sim \partial_{\mu} \mu^{q-1}=(q-1) \mu^{q-2}$ that is singular at $\mu=0$ for anisotropic scattering $(1<q<2)$.

In order to generalize the stochastic method for $q \geqslant 1$, note that the singularity is removed by applying Itô's formula to $\mu^{2-q}$ :

$$
d \mu^{2-q}=(2-q) \mu^{1-q} d \mu+(2-q)(1-q) \mu^{-q} D_{\mu \mu} d t .
$$

Eliminating $d \mu$ between Equations (6) and (9) gives

$$
\begin{aligned}
\frac{1}{(2-q)} \mu^{q-1} d \mu^{2-q}= & {\left[\frac{v}{2 L}\left(1-\mu^{2}\right)-\frac{2 \mu}{1-\mu^{2}} D_{\mu \mu}\right] d t } \\
& +\sqrt{2 D_{\mu \mu}} d W .
\end{aligned}
$$

It is now clear that a Smoluchowski-type approximation for an arbitrary $q \geqslant 1$ corresponds to setting $d \mu^{2-q}=0$, so that the right-hand side of Equation (10) vanishes. Using the resulting equation to express $\mu d t$ gives

$$
\mu d t=\frac{v}{4 L} \frac{\left(1-\mu^{2}\right)^{2}}{D_{\mu \mu}} d t+\frac{\left(1-\mu^{2}\right)}{\sqrt{2 D_{\mu \mu}}} d W,
$$

which is substituted into Equation (5) to yield

$$
d z=\frac{v^{2}}{4 L} \frac{\left(1-\mu^{2}\right)^{2}}{D_{\mu \mu}} d t+\frac{v\left(1-\mu^{2}\right)}{\sqrt{2 D_{\mu \mu}}} d W .
$$

This is a stochastic equation for diffusive particle transport. The first term on the right-hand side of Equation (12) describes coherent streaming and the second term describes diffusion.

Averaging Equation (12) over $\mu$ and rewriting it as an equivalent partial differential equation for the density $F=$ $1 / 2 \int_{-1}^{1} f d \mu$ gives

$$
\frac{\partial F}{\partial t}+u \frac{\partial F}{\partial z}=\kappa_{\|} \frac{\partial^{2} F}{\partial z^{2}} .
$$


Here

$$
\kappa_{\|}=\frac{v^{2}}{4}\left\langle\frac{\left(1-\mu^{2}\right)^{2}}{D_{\mu \mu}}\right\rangle_{h}
$$

is the parallel diffusion coefficient, and

$$
u=\frac{\kappa_{\|}}{L}
$$

is the coherent speed. Here and in what follows, the angular brackets denote the averaging over the relaxed pitch-angle distribution $h(\mu)$, given by Equation (7):

$$
\langle\phi\rangle_{h}=\frac{\int_{-1}^{1} \phi e^{G} d \mu}{\int_{-1}^{1} e^{G} d \mu} .
$$

The subscript $h$ is a reminder that the averaging is not just integration over $\mu$ as in Beeck \& Wibberenz (1986). Now integration of Equation (14) by parts gives simple, physically transparent expressions for the transport coefficients:

$$
\begin{gathered}
\kappa_{\|}=v L\langle\mu\rangle_{h}, \\
u=v\langle\mu\rangle_{h} .
\end{gathered}
$$

Equations (13), (17), and (18) constitute the diffusion approximation to focused cosmic-ray transport. The equations agree with Equations (14) and (17) in Beeck \& Wibberenz (1986). The sign of the convective term in Equation (13) is different from that in Equation (17) in Beeck \& Wibberenz (1986). This is because Equation (13) is expressed in terms of the linear density $f$ (the number of particles per line of force per unit distance parallel to $B_{0}$ ) rather than in terms of the phase-space density $f_{0}$ as in Beeck \& Wibberenz (1986). The two descriptions are mathematically equivalent, and the choice of $f$ or $f_{0}$ is only a matter of convenience (Earl 1981).

As a concrete illustration of effects of helicity on particle transport, consider the case of helicity-modified isotropic scattering when complete analytical treatment is possible. Using Equation (4) with $q=1$ and $e B_{0}>0$ in Equation (17) yields

$$
\begin{aligned}
& \frac{\kappa_{\|}}{v L}= \\
& \frac{\left(1-\frac{1+\sigma}{\xi}\right)(1+\sigma) \exp \left(\frac{\xi}{1+\sigma}\right)+\left(1+\frac{1-\sigma}{\xi}\right)(1-\sigma) \exp \left(-\frac{\xi}{1-\sigma}\right)+4 \frac{\sigma}{\xi}}{(1+\sigma) \exp \left(\frac{\xi}{1+\sigma}\right)-(1-\sigma) \exp \left(-\frac{\xi}{1-\sigma}\right)-2 \sigma} .
\end{aligned}
$$

Here $\xi=\lambda_{0} / L$ is a parameter quantifying the focusing strength and $\lambda_{0}=v /\left(2 D_{0}\right)$ is the scattering mean free path in the absence of focusing and helicity. This expression generalizes Equation (16a) in Beeck \& Wibberenz (1986), which is valid when the net polarization vanishes $(\sigma=0)$ :

$$
\frac{\kappa_{\|}}{v L}=\operatorname{coth} \xi-\frac{1}{\xi} \text {. }
$$

Two other limiting cases of Equation (19) are of interest. In the absence of focusing ( $L \rightarrow \infty$ or $\xi \rightarrow 0$ ), Equation (19) yields

$$
\kappa_{\|}=\frac{1}{\left(1-\sigma^{2}\right)} \frac{v^{2}}{6 D_{0}},
$$

which contains the familiar factor $\left(1-\sigma^{2}\right)$ quantifying the effect of helicity on the diffusion coefficient in a uniform magnetic field (Earl 1992). In the opposite limit of strong focusing $(L \rightarrow 0$ or $\xi \rightarrow \infty$ ), Equation (19) reduces to

$$
\kappa_{\|}=v L
$$

and so $u=\kappa_{\|} / L=v$. Hence the focusing length $3 L$ plays the role of an effective scattering mean free path in the limit $\xi \rightarrow \infty$. Interestingly, this appears to be a sensible result even though the diffusion approximation is usually expected to be accurate only for $\xi<1$, so that the particle pitch-angle distribution is close to the isotropic distribution.

The reader may amuse him/herself with a much harder case, $q=1.5$, which should also allow integration of Equation (17) in elementary functions. For an arbitrary $q$, a series expansion for $\kappa_{\|}$can be written down if focusing is weak enough. After some algebra, Equation (17) yields the parallel diffusion coefficient with account taken of first-order terms in $\lambda_{0} / L$ :

$$
\begin{aligned}
\kappa_{\|}= & \frac{\lambda_{0} v}{3\left(1-\sigma^{2}\right)}\left(1-\frac{\sigma}{\left(1-\sigma^{2}\right)} \operatorname{sgn}\left(e B_{0}\right)\right. \\
& \left.\times \frac{(2-q)(4-q)}{6(3-q)} \frac{\lambda_{0}}{L}+\cdots\right)
\end{aligned}
$$

(cf. Equations (40) and (51) in Bieber \& Burger 1990). Here

$$
\lambda_{0}=\frac{3 v}{8} \int_{-1}^{1} \frac{\left(1-\mu^{2}\right)^{2}}{D_{\mu \mu}} d \mu=\frac{3}{2(2-q)(4-q)} \frac{v}{D_{0}}
$$

is the scattering mean free path in the absence of focusing $(L=\infty)$ and helicity $(\sigma=0)$ (Jokipii 1966; Hasselmann \& Wibberenz 1970).

It is worth stressing that the present analysis is valid for arbitrary $\sigma$ and $L$. It shows that the general expression for the parallel diffusion coefficient $\kappa_{\|}$in terms of $D_{\mu \mu}$, given by Equation (14) in Beeck \& Wibberenz (1986), remains valid for the helicity-modified scattering rate of Equation (4). The formula for $\kappa_{\|}$(Equation (17) above), however, differs from the expressions given by Equation (18) in Bieber \& Burger (1990) or Equation (52) in Kóta (2000), except in the limiting case $\sigma=0$. It does not mean that either expression is incorrect, yet the assumptions on which each formula is based need to be understood.

Bieber \& Burger (1990) argued that the central assumption made in the analysis by Beeck \& Wibberenz (1986) is that the spatial scale of the cosmic-ray anisotropy is given by the focusing length $L$, whereas the set of transport parameters in Bieber \& Burger (1990) applies when the cosmic-ray parallel streaming vanishes. An alternative, perhaps more physically transparent point of view follows from the analysis of this paper. The diffusion approximation of Beeck \& Wibberenz (1986) is a consequence of strong scattering that causes the cosmic-ray angular distribution to be closely approximated by the steady distribution of Equation (7). Either way, the assumption that the relevant spatial scale is much greater than $L$ is more likely to be valid in the context of the solar modulation of galactic cosmic rays rather than for the propagation of solar energetic particles (Kóta 2000). Hence the diffusion model of Beeck \& Wibberenz (1986) and the present paper is better suited to the analysis of solar energetic particles than the diffusion model developed by Bieber \& Burger (1990) and Kóta (2000).

Finally, note that Schlickeiser \& Shalchi (2008) derived a general cosmic-ray diffusion approximation, including the effects of the magnetic and cross helicities on the diffusion 
coefficients. Schlickeiser \& Shalchi (2008), however, assumed from the outset that focusing is weak, $\lambda_{0} / L \ll 1$, which is why their expression for $\kappa_{\|}$is independent of $L$ and their analysis can only be compared with the present results in the limit $L \rightarrow \infty$. When a typographic error is corrected in Equation (24) of Schlickeiser \& Shalchi (2008), it gives the dependence of $\kappa_{\|}$on $H_{c}, \sigma^{-}$, and $\sigma^{+}$, which reduces to the correct behavior $\kappa_{\|} \sim\left(1-\sigma^{2}\right)^{-1}$ in the limit $H_{c}=1$ and $\sigma^{-}=0$.

\section{DISCUSSION}

Techniques of stochastic differential equations can be applied to a wide range of problems in cosmic-ray transport (e.g., MacKinnon \& Craig 1991; Krülls \& Achterberg 1994; Conway et al. 1998; Litvinenko 2009). As illustrated in this paper, the diffusive approximation for the propagation of solar energetic particles in the interplanetary space can be derived in a straightforward and elegant way if the Fokker-Planck equation is replaced by the equivalent stochastic equations that are simplified using a Smoluchowski-type approximation.

This paper emphasizes the key assumption of rapid relaxation of the angular particle distribution to a non-isotropic quasisteady distribution in the presence of adiabatic focusing. Depending on the parameter regime, different sets of transport parameters may be appropriate in different applications. This simple fact is reflected in the assumptions made when the transport coefficients are calculated. The present approach supplements the previous classification of the parameter regimes in terms of the magnitude of the particle density gradient (Bieber \& Burger 1990; Kóta 2000).

Independent of the calculation method adopted, the previous studies of the problem, as well as this paper, lead to the same expression for the parallel diffusion coefficient $\kappa_{\|}$of solar energetic particles in the limit of vanishing net polarization $\sigma$ (Earl 1981; Beeck \& Wibberenz 1986; Bieber \& Burger 1990; Kóta 2000). Shalchi (2011), however, presented a formula for $\kappa_{\|}$that differs from all the others. Litvinenko (2012) analyzed in detail the case of isotropic scattering $(q=1)$ and argued that Shalchi (2011) had erroneously postulated the complete loss of memory of the initial angular distribution. This paper generalizes the argument in Litvinenko (2012) to an arbitrary $q$ and thus shows that the mistake in Shalchi (2011) is not limited to the isotropic scattering case. I am disappointed to observe that He \& Wan (2012) adopted the erroneous results of Shalchi (2011) in their calculation of the parallel mean free path of solar energetic particles.

Given the usefulness of the diffusion approximation for interpretation of solar energetic particle observations (e.g., Litvinenko \& Schlickeiser 2011; Artmann et al. 2011), it would be interesting to generalize it by calculating the transport coefficients as functions of the cross helicity parameter $-1 \leqslant$ $H_{c} \leqslant 1$ and both polarization parameters $\sigma^{-}$and $\sigma^{+}$in the presence of adiabatic focusing. So far such a calculation has been performed only in the weak focusing limit (Schlickeiser \& Shalchi 2008). Although the present approach relaxes the weak focusing assumption, the results have been presented for the slab model of turbulence with waves propagating with one phase speed only, in which case the helicity-modified scattering coefficient $D_{\mu \mu}$ in the Fokker-Planck equation depends on a single polarization parameter (Goldstein \& Matthaeus 1981). Analysis of a more general model for scattering (Dung \& Schlickeiser 1990) may be required to interpret features of flareaccelerated particles observed in interplanetary space.

The diffusion approximation of cosmic-ray transport is simple and convenient, but it has well-known limitations. Perturbation techniques or series expansions are employed to obtain a more accurate description of particle propagation. Those methods lead to a modified telegrapher's equation for particle density, which makes it possible to describe the propagation of a localized coherent pulse and reveals the dependence of the coherent speed on the magnetic helicity (Earl 1992; Gombosi et al. 1993; Pauls \& Burger 1994). It would be interesting to explore whether higher-order corrections to the Smoluchowski approximation could be developed which would lead to comparable results.

This work was partially supported by NASA through a grant to the University of New Hampshire (USA) and by the Deutsche Forschungsgemeinschaft through a grant to the DFGForschergruppe 1048 at Ruhr-Universität Bochum (Germany).

\section{REFERENCES}

Artmann, S., Schlickeiser, R., Agueda, N., Krucker, S., \& Lin, R. P. 2011, A\&A, 535, A92

Beeck, J., \& Wibberenz, G. 1986, ApJ, 311, 437

Bieber, J. W., \& Burger, R. A. 1990, ApJ, 348, 597

Bieber, J. W., Dörge, W., Evenson, P. A., et al. 2002, ApJ, 567, 622

Bieber, J. W., Evenson, P., \& Matthaeus, W. H. 1987, Geophys. Res. Lett., 14, 864

Conway, A. J. 2000, A\&A, 362, 383

Conway, A. J., MacKinnon, A. L., Brown, J. C., \& McArthur, G. 1998, A\&A, 331,1103

Dung, R., \& Schlickeiser, R. 1990, A\&A, 237, 504

Earl, J. A. 1981, ApJ, 251, 739

Earl, J. A. 1992, ApJ, 395, 185

Fichtner, H., le Roux, J. A., Mall, U., \& Rucinski, D. 1996, A\&A, 314, 650

Gardiner, C. W. 2009, Stochastic Methods: A Handbook for the Natural and Social Sciences (Berlin: Springer)

Goldstein, M. L., \& Matthaeus, W. H. 1981, in Proc. 17th Int. Cosmic Ray Conf., Paris (Gif-sur-Yvette, France: CEA), 3, 294

Gombosi, T. I., Jokipii, J. R., Kota, J., Lorencz, K., \& Williams, L. L. 1993, ApJ, 403, 377

Hasselmann, K., \& Wibberenz, G. 1968, Z. Geophys., 34, 353

Hasselmann, K., \& Wibberenz, G. 1970, ApJ, 162, 1049

He, H.-Q., \& Wan, W. 2012, ApJ, 747, 38

Jokipii, J. R. 1966, ApJ, 146, 480

Kóta, J. 2000, J. Geophys. Res., 105, 2403

Krülls, W. M., \& Achterberg, A. 1994, A\&A, 286, 314

Litvinenko, Y. E. 2009, A\&A, 496, 129

Litvinenko, Y. E. 2012, ApJ, 745, 62

Litvinenko, Y. E., \& Schlickeiser, R. A. 2011, ApJ, 732, L31

MacKinnon, A. L., \& Craig, I. J. D. 1991, A\&A, 251, 693

Meyer, P., Parker, E. N., \& Simpson, J. A. 1956, Phys. Rev., 104, 768

Pauls, H. L., \& Burger, R. A. 1994, ApJ, 427, 927

Pei, C., Bieber, J. W., Burger, R. A., \& Clem, J. 2010, J. Geophys. Res., 115, A12107

Roelof, E. C. 1969, in Lectures in High Energy Astrophysics, ed. H. Ögelman \& J. R. Wayland (NASA SP-199; Washington, DC: NASA), 111

Sáiz, A., Ruffolo, D., Bieber, J. W., Evenson, P., \& Pyle, R. 2008, ApJ, 672, 650 Schlickeiser, R. 1989, ApJ, 336, 243

Schlickeiser, R. 2011, ApJ, 732, 96

Schlickeiser, R., \& Shalchi, A. 2008, ApJ, 686, 292

Shalchi, A. 2011, ApJ, 728, 113

Strauss, R. D., Potgieter, M. S., Büsching, I., \& Kopp, A. 2011, ApJ, 735, 83

Zhang, M. 1999, ApJ, 512, 409 